\title{
Identification of microRNAs expressed in the midgut of Aedes albopictus during dengue infection
}

\author{
Jianxin Su ${ }^{1,2}$, Chunxiao Li ${ }^{1}$, Yingmei Zhang ${ }^{1}$, Ting Yan ${ }^{1}$, Xiaojuan Zhu', Minghui Zhao ${ }^{1}$, Dan Xing ${ }^{1}$, Yande Dong ${ }^{1}$, \\ Xiaoxia Guo ${ }^{1 *}$ and Tongyan Zhao ${ }^{1 *}$
}

\begin{abstract}
Background: The midgut is the first barrier to dengue virus (DENV) infections of mosquitoes and therefore is a major bottleneck for the subsequent development of vector competence. However, the molecular mechanisms responsible for this barrier are unknown.

Results: We constructed three small RNA libraries from the midguts of adult Aedes albopictus females that had been fed on either sugar solution, an uninfected blood meal, or a blood meal infected with DENV-2, and112 conserved microRNAs represented by 173 miRNA sequences were identified, with 34 novel microRNAs predicted by Mireap, RNAfold and Sfold software. In addition, the expression of aal-miR-1174, aal-miR-2951 and aal-miR-956 was confirmed via stem-loop quantitative real-time PCR (qRT-PCR). Compared with microRNA expression profiles of mosquitoes that had ingested a regular blood meal, 43 microRNAs were upregulated and 4were downregulated in mosquitoes that had ingested a DENV-2-infected blood meal. Among the differentially expressed microRNAs, miR-1767, miR-276-3p, miR-4448 and miR-4728-5p were verified via stem-loop qRT-PCR.

Conclusions: Analyses indicated that the changing patterns in miRNA expression during DENV-2 infection were significant and varied at different time points post infection. Most miRNA were upregulated at $24 \mathrm{~h}$ but were downregulated at $48 \mathrm{~h}$ post DENV-2 intake. The aal-miR-4728-5p was chosen for an in vitro transient transfection assay, and the results show that this miRNA enhances DENV replication in C6/36 cells. This study provides the first information on microRNAs expressed in the midgut of Ae. albopictus and describes species-specific changes in their expression levels following infection by DENV-2.
\end{abstract}

Keywords: Aedes albopictus, MicroRNA (miRNA), Midgut, Dengue virus (DENV)

\section{Background}

The dengue virus (DENV) is one of the most common causes of vector-borne viral disease in tropical and subtropical areas [1, 2]. Approximately 50 million dengue infections occur every year, and at least 2.5 billion people are estimated to be at risk of dengue-related diseases worldwide [3]. Aedes aegypti L. and Ae. albopictus (Skuse) are the principal vectors of the DENV [4]. Because a dengue fever (DF) vaccine has yet to be developed, vector control is currently the only effective means

\footnotetext{
*Correspondence: guoxx99@163.com; tongyanzhao@126.com

${ }^{1}$ State Key Laboratory of Pathogen and Biosecurity, Beijing Institute of

Microbiology and Epidemiology, Beijing 100071, People's Republic of China

Full list of author information is available at the end of the article
}

of preventing this disease $[3,5]$. However, despite efforts focused on vector control, the global pandemic of DF has increased dramatically in recent decades because of increases in vector and human population densities, which have been accompanied by a sharp increase in more severe manifestations of the disease, and therefore, alternative control strategies are being investigated. Some of these efforts have been made based on the genetic manipulation of insect vectors to modulate characteristics such as vector competence [6-8], but the manipulation of vector competence must be based on extensive knowledge of the molecular factors underlying vector-pathogen interactions. 
Vector competence is the intrinsic ability of arthropod vectors to acquire, maintain, and transmit a pathogen. Several barriers exist in mosquitoes that can hinder the infection, dissemination, and transmission of arbovirus, including DENVs $[9,10]$. Of these, the midgut is the first barrier to the invasion of pathogens ingested into the alimentary tract. The ability of midgut epithelial cells to resist viral infection is the main factor determining the susceptibility of mosquitoes to arbovirus infections and a key index of vector competence $[11,12]$. Currently, the molecular mechanisms underlying the specific binding between viral pathogens and midgut epithelial cells that regulate viral replication are still unclear. This in turn has been an obstacle to research on the molecular mechanisms responsible for the susceptibility of mosquito vectors to DENV. Understanding the molecular mechanisms underlying these differences in vector competence is important in assessing the risks posed by any particular arbovirus, assessing mosquito vector combinations and indeveloping novel strategies to mitigate or block the transmission of mosquito-borne arboviruses.

MicroRNAs (miRNAs) are a class of non-coding RNAs that regulate gene expression at the post-transcriptional level $[13,14]$. Several studies have shown that miRNAs play important roles in controlling viral infections and conferring innate immunity $[15,16]$. In Anopheles gambiae, Dcr1, Dcr2 and Drosha transcripts have been shown to exhibit enhanced associations with polysome in the midgut after Plasmodium infection [17], and knocking down Dcr1 and Ago1 mRNAs in the midgut of this species led to an increased susceptibility to Plasmodium infections [18]. Transgenic Ae. aegypti mosquitoes in which Aa-dcr2 gene expression in the midgut had been suppressed,show significantly higher Sindbis virus titres, midgut infection rates and virus dissemination rates than normal mosquitoes [12]. In Ae aegypti, a transgenic family that expressed an inverted-repeat RNA in the midgut was constructed that displayed a remarkable reduction in DENV-2 replication in the midgut after ingesting blood meals infected with DENV-2 [19]. These experiments demonstrate that miRNAs play important roles in the process of pathogen infection in mosquitoes and can affect the sensitivity of the midgut to DENV infection. They also provide a theoretical basis for the genetic manipulation of vector competence.

Currently, most studies on miRNAs in Aedes mosquitoes have focused on Ae. aegypti, in which at least 88 miRNAs have been identified (miRBase 20, http:// www.mirbase.org/). Several studies have shown that DENV infection causes changes in miRNA expression in the midgut of this species, some of which enhance DENV infections in cultured cell lines [20, 21]. Although Ae. albopictus and Ae. aegypti both belong to the subgenus Stegomyia [22], Ae. aegypti is generally a more efficient disease vector but is less susceptible to DENV infections than Ae. albopictus. In general, more susceptible species can become infected by a lower viral load than less susceptible species, which suggests that Ae. albopictus could also function as a maintenance vector during interepidemic periods [23]. Several studies have demonstrated that the midgut of Ae. albopictus is more susceptible to DENV infection than that of Ae. aegypti, but the subsequent dissemination of DENV from the midgut is slower in Ae. albopictus [24-26], thus the miRNA involving in midgut infection by DENV should be different. These results suggest the existence of interspecific differences in both vector competence and the molecular mechanisms responsible for the midgut invasion barrier [27].

Although some Ae. albopictus miRNAs have previously been identified, none were midgut miRNAs. Many miRNAs have distinct expression patterns in different organs, and some areeven tissue-specific [28]. For example, miR1175, miR-1174, miR-281 and miR-989 are only expressed in the midgut of $A n$. gambiae, and the expression of miR989 is restricted in females. MiR-12 and miR-283 are expressed in the gut and thorax of An. gambiae and in the foregut, posterior midgut, hindgut and salivary glands of Drosophila melanogaster embryos [29]. These tissuespecific expression patterns of some miRNAs imply that they have different roles in different organs. Because the midgut is the first barrier to a DENV infection [11, 12], identifying the patterns and potential roles of different midgut miRNAs during the course of a DENV infection could aid in the identification of the molecular mechanisms responsible for the midgut infection barrier and thereby facilitating the control of DENV-related diseases. Here, we present the first investigation of midgut miRNAs in Ae. albopictus, including changes in their expression levels during the process of DENV infection in this species.

\section{Methods \\ Mosquito collection and husbandry}

Aedes albopictus mosquitoes were collected from Guangzhou City in 2012 and reared in an insectary under laboratory conditions (temperature $26 \pm 1^{\circ} \mathrm{C}$, relative humidity $80 \%$, light: dark photoperiod $14: 10$ ) to the 5 th $(5 \mathrm{~F})$ generation. Adult mosquitoes were provided with a $10 \%$ glucose solution, and females were allowed to feed on the blood of healthy mice to produce eggs.

Adult female mosquitoes were randomly assigned to three groups 4 to 6 days after emergence. Group $C$ was fed on sugar solutiononly; Group B was fed on uninfected blood meal (blood:glucose solution:brain suspension of normal suckling mice $=1: 1: 1$ ); and Group $D$ was fed on an artificial DENV-2 blood meal (blood:10\% glucose solution: DENV suspension=1:1:1). Mosquitoes were starved for 12-16 h before being allowed to feed for approximately $1 \mathrm{~h}$. Fully engorged mosquitoes were 
selected after cold anaesthetization at $-20{ }^{\circ} \mathrm{C}$ for 2 min and transferred to a separate insectary under the conditions described above. Midguts were dissected from mosquitoes 24-26 h after they had fed.

\section{Virus strains}

DENV-2 virus (New Guinea C strain, NGC) was obtained from the Microbial Culture Collection Center of the Beijing Institute of Microbiology and Epidemiology (Beijing, China). The DENV-2 starting stock was prepared in 1-day-old suckling mice via intracerebral inoculation. A suspension of infected mouse brains was made in Dulbecco's modified Eagle's medium (DMEM, Gibco) and stored at $-70{ }^{\circ} \mathrm{C}$ until use.

\section{Mosquito dissection and RNA extraction}

Midguts from approximately 100 mosquitoes from each of the $\mathrm{C}, \mathrm{B}$ and $\mathrm{D}$ groups were collected by dissecting individual mosquitoes in a drop of cold physiological saline. Midguts from the three groups were stored separately in $1.5-\mathrm{ml}$ RNase-free microcentrifuge tubes with $1.0 \mathrm{ml}$ RNApreserving liquid and then flash frozen and stored at $-80^{\circ} \mathrm{C}$ until subsequent RNA isolation. Total RNAs from the three groups were extracted using the RNeasy Mini Kit (Qiagen,
Beijing, China) according to the manufacturer's instructions. The final total RNA was dissolved in $20 \mu \mathrm{l}$ RNase-free water.

Two sets of samples were prepared; one set was stored at $-80^{\circ} \mathrm{C}$ for future verification, and the other was analysed via electrophoresis on a $15 \%$ denaturing polyacrylamide gel after which small RNAs in the 15-30 nt range were purified and ligated with a 3 ' adapter (5PUCGUAUGCCGUCUUCUG CUUGUidT) and a $5^{\prime}$ adapter(5GUUCAGAGUUCUACA GUCCGACGAUC). These were then reverse transcribed using the primer 5'-CAA GCA GAA GAC GGC ATA CGA-3' and proliferated using forward and reverse primers (5'-AAT GAT ACG GCG ACC ACC GAC AGG TTC A GA GTT CTA CAG TCC GA-3' and 5'-CAA GCA GAA GAC GGC ATA CGA-3'). PCR products were purified via phenol/chloroform extraction and ethanol precipitation and shipped to BGI (Shenzhen, China) for high-throughput deep sequencing.

\section{qRT-PCR}

Stem-loop qRT-PCR analysis was performed using an ABI STEP-ONE PLUS Real Time PCR System (Applied Biosystems). The specific miRNA stem-loop primers were designed by BGI (Shenzhen, China), and the RealTime primers were designed with Primer2.0. All primers are shown in Table 1 . The reverse transcription reaction was

Table 1 Primer sequences used in this study

\begin{tabular}{|c|c|c|}
\hline miRNA name & Primer name & Sequence $\left(5^{\prime}-3^{\prime}\right)$ \\
\hline \multirow[t]{2}{*}{ aal-miR-1767 } & RT-primer & GTCGTATCCAGTGCGTGTCGTGGAGTCGGCAATTGCACTGGATACGACACCTTG \\
\hline & Forward primer & AGACAGGAGAACAGCA \\
\hline \multirow[t]{2}{*}{ aal-miR-276-3p } & RT-primer & GTCGTATCCAGTGCGTGTCGTGGAGTCGGCAATTGCACTGGATACGACGAGCAC \\
\hline & Forward primer & TAGGAACTTCATACCG \\
\hline \multirow[t]{2}{*}{ aal-miR-4448 } & RT-primer & GTCGTATCCAGTGCGTGTCGTGGAGTCGGCAATTGCACTGGATACGACACCCCT \\
\hline & Forward primer & GGCTCGTTGGTCTAGG \\
\hline \multirow[t]{2}{*}{ aal-miR-4728-5p } & RT-primer & GTCGTATCCAGTGCGTGTCGTGGAGTCGGCAATTGCACTGGATACGACTGCTGC \\
\hline & Forward primer & TGGGAGGGCAGAGGGG \\
\hline \multirow[t]{2}{*}{ aal-miR-1174 } & RT-primer & GTCGTATCCAGTGCGTGTCGTGGAGTCGGCAATTGCACTGGATACGACAGTTGG \\
\hline & Forward primer & TCAGATCTAACTAATACCCAA \\
\hline \multirow[t]{2}{*}{ aal-miR-2951 } & RT-primer & GTCGTATCCAGTGCGTGTCGTGGAGTCGGCAATTGCACTGGATACGACTCGCCC \\
\hline & Forward primer & AAGAGCTCAGCACGCAGG \\
\hline \multirow[t]{2}{*}{ aal-miR-956-3p } & RT-primer & GTCGTATCCAGTGCGTGTCGTGGAGTCGGCAATTGCACTGGATACGACAATGAT \\
\hline & Forward primer & TITCGAGACCACTGCAAAT \\
\hline \multirow[t]{2}{*}{ aal-miR-956-5p } & RT-primer & GTCGTATCCAGTGCGTGTCGTGGAGTCGGCAATTGCACTGGATACGACAGTTAA \\
\hline & Forward primer & GTTTGAAATGGTCTCGTTAAC \\
\hline Universal & Reverse primer & GTGCGTGTCGTGGAGTC \\
\hline \multirow[t]{2}{*}{ rpS7 } & Forward primer & ATGGTITCGGATCAAAGGT \\
\hline & Reverse primer & CGACCTTGTGTTCAATGGTG \\
\hline \multirow[t]{2}{*}{ DENV-2 } & Forward primer & TCAATATGCTGAAACGCGCGAGAAACCG \\
\hline & Reverse primer & TTGCACCAACAGTCAATGTCTTCAGGTTC \\
\hline
\end{tabular}

The Probe Library probe binding site is shown in bold type 
performed with the One Step PrimeScript RT Reagent Kit (Qiagen, Beijing, China) according to the manufacturer's protocol. Three replicates were performed for each sample, and the Ae. albopictus house-keeping rpS7 gene was used as an internal reference [30]. The relative expression of each miRNA was calculated using the $2^{-\Delta \Delta}$ CT method [31]. The reaction mixture contained $1.6 \mu \mathrm{MgCl}_{2}$ (TaKaRa), $0.1 \mu \mathrm{l}$ SYBRGEEN (Invitrogen), $2 \mu \mathrm{l} 10 \times$ buffer (TaKaRa), $0.4 \mu \mathrm{l}$ dNTP (10 mM/each; BGI, China), $0.2 \mu \mathrm{l}$ forward and reverse primer $(50 \mathrm{pM} / \mu \mathrm{l}$; BGI, China), $1 \mu \mathrm{l}$ Template (cDNA), $0.1 \mu \mathrm{l}$ ROX (Invitrogen), $0.2 \mu \mathrm{l} \mathrm{Taq} \mathrm{(5U/ \mu l;} \mathrm{Kap-}$ pa,USA) and $14.2 \mu \mathrm{l} \mathrm{H}_{2} \mathrm{O}$ in a final volume of $20 \mu \mathrm{l}$. The qRT-PCR program was $95^{\circ} \mathrm{C}$ for $2 \mathrm{~min}$, followed by $40 \mathrm{cy}$ cles of $94{ }^{\circ} \mathrm{C}$ for $10 \mathrm{~s}, 53{ }^{\circ} \mathrm{C}$ for $10 \mathrm{~s}$ and $72{ }^{\circ} \mathrm{C}$ for $40 \mathrm{~s}$. The melting curves of selected miRNAs were determined after amplification using the following program: $95{ }^{\circ} \mathrm{C}$ for $30 \mathrm{~s}$, and $65{ }^{\circ} \mathrm{C}$ for $15 \mathrm{~s}$, followed by an increase in temperature to $95{ }^{\circ} \mathrm{C}$ while continuously recording the fluorescent signal.

\section{Alignment of conserved miRNAs using BLAST and tag2 miRNA software}

We first mapped all clean small RNA tags by matching them to GenBank rRNA, scRNA, snoRNA, snRNA and tRNA databases and removed matched tags from unannotated tags. To make sure every unique small RNA mapped to only one annotation, we adhered to the following priority rule: all rRNA (in which GenBank $>$ Rfam) $>$ repeat $>$ exon $>$ intron $>$ known miRNA. Because miRBase does not currently contain Ae. albopictus miRNA, we first used BLAST to align small RNA tags with the Ae. aegypti miRNA precursor in miRBase19.0 to obtain a miRNA count with no mismatches. We then aligned tags to all mature animal miRNAs in miRBase19.0 using tag2 miRNA software (developed by BGI; Shenzhen, China). The specific steps we used were as follows: (i) We first matched mature sequences from our clean data to those of animal miRNAs in miRBase19.0, considering interspecific variations and allowing up to two mismatches and a few gaps; (ii) From the miRNA families identified through the above process, we selected miRNA sequences with the highest expression levels among the different species in the same family for a provisional miRNA database; (iii) We then constructed a miRNA database by comparing and aligning the cleandata to known miRNA expression profiles. We calculated expression levels by comparing the clean data to miRNA sequences in the provisional database and by summing the number of those that aligned with up to 2 mismatches. This verification process gave us a high probability of obtaining meaningful results.

\section{Prediction of novel miRNA candidates using Mireap software}

Mireap software was used to predict novel miRNAs by exploring their secondary structure, Dicer cleavage sites and the minimum free energy of unannotated small RNA tags that could be mapped to the Ae. aegypti genome. Mireap can be accessed from the following link: http://sourceforge.net/projects/mireap/. Some key conditions for novel miRNA prediction are as follows: (i) The tags used to predict novel miRNAs were unannotated tags that could be perfectly matched to the intron and antisense exon regions of the reference genome (Ae. aegypti); (ii) Of those genes whose sequences and structures satisfied the above criteria, those with hairpin miRNAs that could enfold secondary structures or mature miRNAs present in one arm of the hairpin precursors were considered as candidate miRNA genes; (iii) Mature miRNA strands and their complementary strand (miRNA*) 2-nucleotide 3' overhangs and (iv) hairpin precursors lacking large internal loops or bulges were considered; ( $v$ ) The secondary structures of the hairpins had to bestable, with a minimum free energy (MFE) lower than or equal to $-20 \mathrm{kcal} / \mathrm{mol}$; and (vi) the number of mature miRNAs with predicted hairpins must have been no less than 5 after alignment.

RNAfold (http://rna.tbi.univie.ac.at/cgi-bin/RNAfold.cgi) was used to screen novel miRNA candidates that satisfied criteria ii-v mentioned above. For those structures predicted by RNA fold for which MFE was $>-25$, Sfold (http://sfold.wadsworth.org/cgi-bin/srna.pl) was used to determine if these were novel miRNAs according to criteria ii-v above.

\section{Analysis of variations in miRNA expression}

To compare the miRNA expression levels in groups $\mathrm{C}, \mathrm{B}$ and $\mathrm{D}$ via high through put deep sequencing, we normalized the read numbers in each library according to the following formula: Normalized expression $=\frac{\text { Actual miRNA reads }}{\text { Total count of clean reads }} \times 10^{6}$. We then calculated the ratio and the magnitude of between-group differences and their associated $P$-values from the normalized data. The ratio was calculated according to the following formula: ratio $=\frac{\text { normalized expression of treatment group }}{\text { normalized expression of control group }}$, and the magnitude of differences is expressed as "fold-change", which was calculated using the following the formula: foldchange $=\log _{2}$ ratio. A fold-change $>1$ or $<-1$ with a $P$ value $<0.05$ was regarded as being significantly different [32]. Fold-changes and their associated $P$-values were calculated using a special procedure developed by the BGI biotech company (Shenzhen, China).

\section{Cell culture and cell infection}

Aedes albopictus C6/36 cells were cultured in RPIM 1640 (Gibco) culture medium supplemented with 10\% heat-inactivated foetalbovine serum (FBS, Gibco) and maintained at $28{ }^{\circ} \mathrm{C}$ without $\mathrm{CO}_{2}$. To establish DENV-2 infections, $\mathrm{C6} / 36$ cells were seeded in 12-well plates to a 
density of approximately $80 \%$. DENV-2 (NGC) at a 0.01 multiplicity of infection (MOI) was diluted in the above growth medium and added to the cells. After being rocked at room temperature for $1 \mathrm{~h}$, the plates were incubated at $37^{\circ} \mathrm{C}$ for $72 \mathrm{~h}$.

\section{Transient transfection of miRNA oligonucleotides}

C6/36 cells were transfected with $50 \mathrm{nmol}$ of an aal-mir4728-5p mimic or inhibitor and negative controls for the mimic $(\mathrm{NCm})$ and inhibitor $(\mathrm{NCi})$ with Lipofectamine 2000 (Invitrogen) according to the manufacturer's recommendation. The cells were inoculated with the DENV-2 inoculum $24 \mathrm{~h}$ post-transfection. qRT-PCR analysis was used to detect the expression levels of aal-mir-4728-5p in C6/36 cells $24 \mathrm{~h}$ and $72 \mathrm{~h}$ post-transfection and to detect the DENV-2 levels in all C6/36 cells $72 \mathrm{~h}$ postinoculation. The oligonucleotides used in this study are listed in Table 2. All miRNA oligonucleotides were purchased from Genepharma (Shanghai, China).

\section{rpS7 q-RTPCR}

The Ae. albopictus house-keeping $\mathrm{rpS7}$ gene was used as an internal control for the qRT-PCR results. The forward primer sequence was 5'-ATG GTT TTC GGA TCA AAG GT-3', and the reverse sequence was 5'-CGA CCT TGT GTT CAA TGG TG-3'. qRT-PCR analyses followed the protocols described above.

\section{Statistical analysis}

A Poisson distribution was used to analyse adigital transcript of the profile data following the method described by Audic \& Claverie [32]. The $2^{-\triangle \Delta}$ CT method was used to determine relative expression levels from the qRTPCR results, and paired $t$-tests were used to determine if the differences were statistically significance using SPSS19.0.

\section{Results}

\section{Overview of the dataset}

To identify miRNAs expressed in the midgut of Ae. albopictus and to explore their functions following the ingestion of DENV-infected blood, three small libraries of Ae. albopictus midgut RNAs were constructed, one from mosquitoes that had been fed on sugar solution $(\mathrm{C})$, one from those that had been fed on uninfected blood (B) and one from those that had been fed on blood containing DENV-2 (D). All three libraries were sequenced via high throughput sequencing, giving totals of 19,709,476, 14,667,037 and 16,085,156 reads from the three groups, respectively. When the three libraries were combined, total read numbers for different lengths of RNA peaked at approximately $21 \mathrm{nt}$. The distribution of 21-nt sRNAs in C, B and D was approximately 18.81, 16.78 and $31.8 \%$, respectively (Fig. 1). A group of 27 -nt clean reads also accounted for a relatively high proportion $(16.28,8.38$ and $4.17 \%$, respectively) of the total RNAs in the three libraries; we suspect that these could be piwi-interacting RNAs (piRNAs).

After removal of the adaptor, insert, poly (A) tail and short RNAs < $18 \mathrm{nt}, 19,444,780,14,376,865$ and $15,762,225$ clean reads were obtained from the C, B and D libraries, respectively. Among the clean reads, miRNA reads accounted for 23.9, 9.17 and $12.25 \%$ of the total, and unannotated, redundant reads accounted for 67.36, 47.72 and $51.57 \%$ of the C, B and D libraries, respectively.

Because a complete Ae. albopictus genome is currently unavailable, we compared the reads obtained to the published Ae. aegypti genome (www.vectorbase.org). Although Ae. albopictus and Ae. aegypti belong to the same subgenus, only approximately $5-8 \%$ of the RNAs in the three libraries matched that of Ae. aegypti (Table 3). Therefore, the majority of the RNAs in each library remain unidentified.

\section{Conserved and novel miRNAs in the midgut of Ae. albopictus}

By comparing the three libraries with miRBase19.0, we identified 112 conserved miRNAs represented by 173 miRNA sequences (Additional file 1: Table S1), the majority of which are conserved in other insects, such as D. melanogaster and An. gambiae, and 71 of these conserved miRNAs had been previously reported in Ae. aegypti. However, we did not find five miRNAs that had been previously reported in Ae. albopictus [33, 34]: aalmiR-286, aal-miR-309, aal-miR-315, aal-miR-929 and aal-miR-971. Notably, aal-miR-1174, which had not previously been detected in Ae. albopictus via northern blotting [35], and aal-miR-2951, which had only previously been reported in Culex quinquefasciatus [33] were sequenced in this experiment. Apart from a few mismatches outside the seed region, the sequences of these were perfect matches to cqu-miR-2951 and aae-miR-

Table 2 Oligonucleotide sequences used in this study

\begin{tabular}{lll}
\hline Name & Sense (5'-3') & Antisense (5'-3') \\
\hline aal-mir-4728-5p mimics & UGGGAGGGCAGAGGGGCAGCA & CUGCCCCUCUGCCCUCCCAUU \\
Negative control for mimic $(\mathrm{NCm})$ & UUCUCCGAACGUGUCACGUTT & ACGGACACGUUCGGAGAATT \\
aal-mir-4728-5p inhitior & UGCUGCCCCUCUGCCCUCCCA & \\
Negative control for inhibitor (NCi) & CAGUACUUUUGUGUAGUACAA & \\
\hline
\end{tabular}




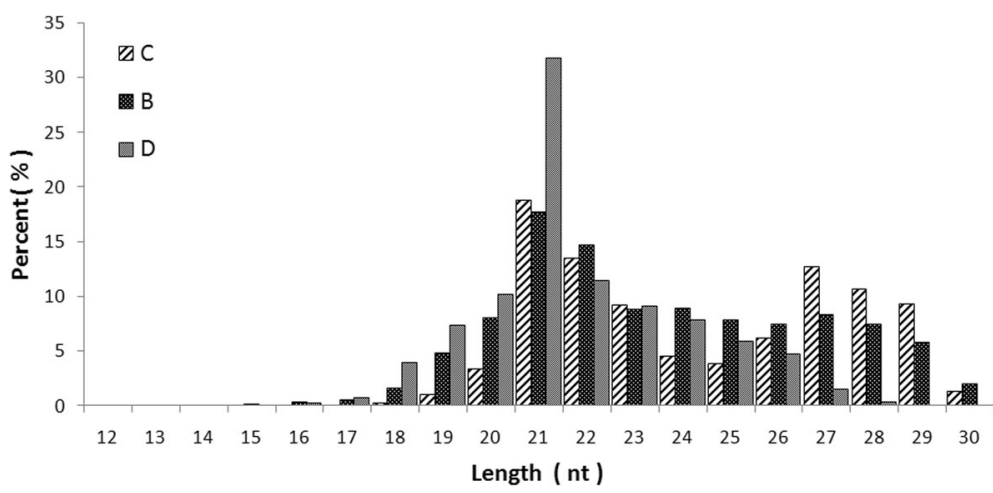

Fig. 1 Frequency of the length of small RNAs in the midguts of Aedes albopictus mosquitoes fed on sugar solution (C), non-infected blood-meal (B) and blood-meal infected with DENV-2 (D)

1174, respectively (miRBase 19.0; Fig. 2b), and their expression was subsequently verified via stem-loop qRTPCR (Fig. 2a, c).

In addition to the conserved miRNAs, a total of 34 novel miRNA candidates were also predicted (Table 4) using Mireap, RNAfold and Sfold. Of these 34 novel miRNA candidates, 2 were expressed after the ingestion of a blood-meal (aal-miR-new33-5p and aal-miR-3960$5 \mathrm{p}$ ), and the others were expressed before the ingestion of a blood meal.

\section{miRNA expression levels in the midgut of sugar-fed $A e$. albopictus}

The normalized data indicate that the expression levels of most miRNAs were low (Additional file 2: Table S2). We divided miRNA sequences in the $C$ group (sugar-fed group) into five classes according to their normalized reads, i.e. $<10 ; 10-10^{2} ; 10^{2}-10^{3} ; 10^{3}-10^{4}$; and $10^{4}-10^{5}$, which were designated Class 1 to Class 5 . Class 1 $(<10)$ contained 68miRNAs, Class $2\left(10-10^{2}\right)$ contained 49 miRNAs, Class $3\left(10^{2}-10^{3}\right)$ contained 36 miRNAs, and Class $4\left(10^{3}-10^{4}\right)$ contained 15 miRNAs that were abundantly expressed, such as miR-317, miR-2940-5p, miR-275-3p, miR-5706. Class $5\left(10^{4}-10^{5}\right)$ contained the remaining 5 miRNAs, which were highly expressed in the midgut: miR-956-3p, miR-184, miR-1-3p, miR-34-5p and miR-281-5p. Of these, aal-miR-956-3p was highly expressed in the midgut; aal-miR-956-5p was also sequenced, but its expression level was very low. The sequences of these two miRNAs matched those of dme-
miR-956-3p and dme-miR-956-5p, respectively, in D. melanogaster (Fig. 2b) and their expression was verified via stem-loop qRT-PCR (Fig. 2a, c). MiR-956 was previously only known to occur in D. melanogaster [36] in miRBase19.0, and its function remains unknown. The Ae. albopictus midgut also shares four of the ten most frequently detected miRNAs in the C7/10 cell line and Cx. quinquefasciatus [33], namely, miR-184, miR-317, miR-275 and miR-8.

\section{Changes in miRNA expression profiles following the ingestion of DENV-infected and non-infected blood} We compared the normalized abundances of miRNAs by calculating the expression ratio of each miRNA between each of the three treatment groups (Fig. 3a-c). Significant differences in miRNA expression were apparent between all three groups, especially in Groups B and D compared with Group C (Fig. 3a, b). On the basis of the foldchanges and the associated $P$-values calculated for each miRNA, 96 miRNAs were downregulated, and 30 were upregulated in GroupB when compared with Group C. Most of the miRNAs that were highly expressed in Group C, such as miR-956-3p, miR-184, miR-1, miR-34, miR281, miR-317, let-7, miR-2945, miR-8, miR-71, miR-275, miR-8, miR-1174, miR-1175, miR-989, miR-998, miR2941, miR-283 and miR-12, were downregulated in Groups B and D. Among the miRNAs that were upregulated, some, such as miR-622, miR-1767, miR-4448, miR3809, miR-3888-5p and miR-2951, were also abundantly expressed before the ingestion of a blood meal, but the

Table 3 Numbers of unique and total sRNAs in the midguts of Ae. albopictus that matched to the Ae. aegypti genome

\begin{tabular}{lllll}
\hline Group & Unique sRNAs & Number matched (\%) & Total sRNAs & Number matched (\%) \\
\hline C & $1,555,751$ & $74,170(4.77)$ & $19,444,780$ & $5,295,808(27.24)$ \\
B & $1,187,330$ & $92,588(7.8)$ & $14,376,865$ & $5,321,300(37.01)$ \\
D & $1,159,505$ & $87,572(7.55)$ & $15,762,225$ & $6,044,644(38.35)$
\end{tabular}

Group C was fed on a sugar solution, B was fed on uninfected blood and D was fed on DENV-2 blood 


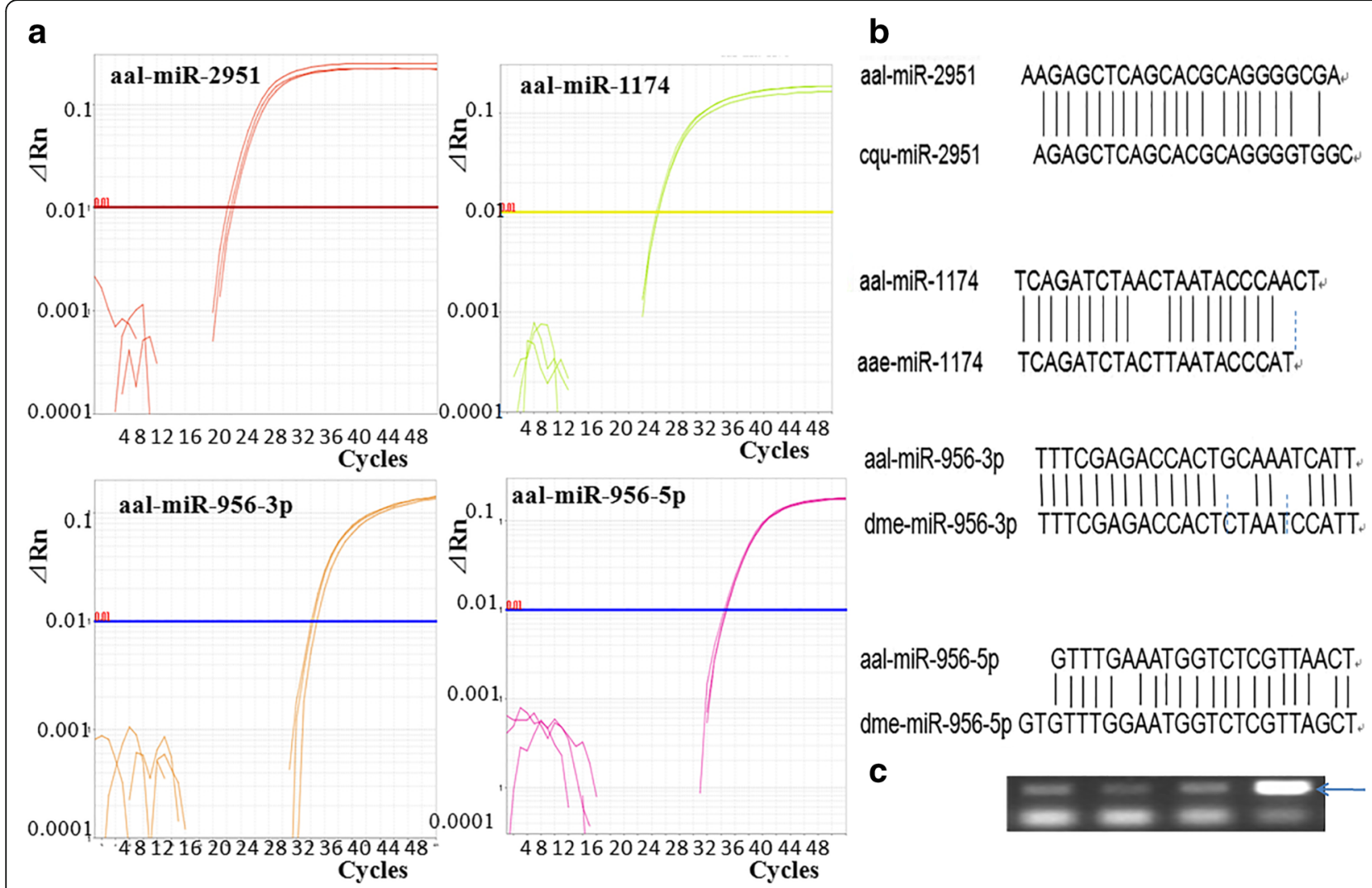

Fig. 2 Verification of four miRNAs in the midgut of Aedes albopictus via stem loop qRT-PCR. a A mplification plots of each miRNA from stem loop qRTPCR results. $\mathbf{b}$ A diagram showing the similarity of each of the four miRNAs to conserved, mature miRNA sequences in miRBase19.0. $\mathbf{c} A$ photograph of the electrophoresis gel ( $4 \%$ agarose) with arrows indicating the size of the expected PCR products corresponding to these four miRNAs

expression of others, such as miR-1951, miR-19c, miR424, miR-103, miR-4728-5p, miR-193-5p, miR-976-5p and miR-3811e-5p, were expressed at low levels before the ingestion of a blood meal, and their expression increased rapidly after the ingestion of a blood meal.

By comparing miRNA expression levels in Group D to those in Group B, we identified 36 miRNAs that were upregulated and 2 that were downregulated in Group D; those with $>2$ - or $<-2$-fold changes in expression between these two groups are listed in Table 5. Aal-miR1767, aal-miR-193-5p, aal-miR-276-3p, aal-miR-4728-5p, aal-miR-622 and aal-miR-4448 all showed significant differences in expression in Group D compared with their expression in Group B (Fig. 4), and the expression levels of these were high.

We chose the four miRNAs that displayed the greatest differences in expression between Groups B and D: aalmiR-1767 (GenBank KY062157), aal-miR-276-3p (GenBank KY062158), aal-miR-4448 andaal-miR-4728-5p (GenBank KY062160), for verification via stem-loop qRT-PCR.

\section{Verification of miRNAs via stem-loop qRT-PCR}

qRT-PCR with a stem-loop primer confirmed the existence of all four of the previously mentioned miRNAs and verified that their expression levels differed significantly between the B and D treatment groups (Fig. 3d). In addition, the trends in their expression levels were consistent with those obtained via high-throughput deep sequencing. We randomly selected aal-miR-4728-5pfor further research.

\section{Enhanced DENV-2 replication in the C6/36 cell line by aal-miR-4728-5p}

Because we had previously confirmed that aal-miR-4728$5 \mathrm{p}$ was also expressed in the Ae. albopictus C6/36 cell line (data not shown), we transfected C6/36 cells with an aal-miR-4728-5p synthetic mimic and inhibitor, as well as negative controls for the mimic $(\mathrm{NCm})$ and inhibitor $(\mathrm{NCi})$, and inoculated them with the DENV-2 virus $24 \mathrm{~h}$ post-transfection. The expression levels of aal-miR4728-5p were measured $24 \mathrm{~h}$ and $72 \mathrm{~h}$ post-transfection, and the expression level of gene for the DENV-2 E protein gene was measured using DENV-2-specific primers (Table 1) $72 \mathrm{~h}$ post-inoculation. qRT-PCR results indicate that the expression of aal-miR-4728-5p increased by 3.09 and 2.41 times $24 \mathrm{~h}$ and $72 \mathrm{~h}$, respectively, after the cells had been transfect with the aal-miR-4728-5p mimic, and its expression was decreased by 0.39 and 0.24 times at the 
Table 4 Novel predicted miRNA candidates in the midgut of Ae. albopictus before and after the ingestion of blood meal using Mireap, RNAfold and Sfold software

\begin{tabular}{|c|c|c|c|c|c|c|c|}
\hline Provisional name & Sequence & Length & Supercontig & Start & End & Strand & Mfe \\
\hline aal-mir-new1-5p & ACGCGGTCGTGCAGGAAATTATT & 23 & 1.1 & $2,315,096$ & $2,315,175$ & + & -39.1 \\
\hline aal-mir-new2-5p & TITGACACTAGAGCGGGGCC & 21 & 1.103 & $2,525,153$ & $2,525,242$ & - & -43.54 \\
\hline aal-mir-new3-3p & TGTTGGAACAGGAGCGGTGACTGG & 24 & 1.1048 & 247,401 & 247,496 & + & -27.2 \\
\hline aal-mir-new4-3p & ACGTGATCTCCCAGCTGGATT & 21 & 1.104 & $2,576,089$ & $2,576,167$ & + & -66.6 \\
\hline aal-mir-new4-5p & TCCAGCTGGGAGATCACGTAC & 21 & 1.104 & $2,576,089$ & $2,576,167$ & + & -66.6 \\
\hline aal-mir-new5-3p & TGAATAAGTGCGGTGAAGACA & 21 & 1.1056 & 241,944 & 242,019 & - & -48.4 \\
\hline aal-mir-new6-5p & TTGAAGGAATCCCTGGAGGCA & 21 & 1.112 & $2,058,675$ & $2,058,759$ & - & -32.1 \\
\hline aal-mir-new7-3p & TGGGGTTGGGCGGAAGGGTTG & 21 & 1.1156 & 159,517 & 159,603 & + & -29.8 \\
\hline aal-mir-new8-5p & ATTCGCACAACAGTCCCATGTT & 22 & 1.125 & $2,091,162$ & $2,091,244$ & + & -28.1 \\
\hline aal-mir-new9-3p & AGAATAAAGACTGCGTAGCCA & 21 & 1.127 & 946,697 & 946,773 & - & -27.7 \\
\hline aal-mir-new10-5p & TTGGCCATTTTGGAACCGGTA & 21 & 1.13 & $1,968,890$ & $1,968,963$ & - & -29.7 \\
\hline aal-mir-new11-5p & TTAAACATAACGTCGGAAGTA & 21 & 1.134 & $1,382,610$ & $1,382,700$ & - & -47.7 \\
\hline aal-mir-new12-5p & TCTGGAGCAACATTTGAAAAG & 21 & 1.163 & 418,620 & 418,704 & + & -24.54 \\
\hline aal-mir-new13-5p & GAATTTGACATTAGAGCGGG & 21 & 1.1642 & 60,969 & 61,064 & + & -59.1 \\
\hline aal-mir-new14-5p & AGAATTTGACACTAGAGCAG & 21 & 1.194 & $1,043,569$ & $1,043,645$ & - & -32.6 \\
\hline aal-mir-new15-5p & TGAAGGAATCCCTGGAGGCAT & 21 & 1.2 & $2,052,582$ & $2,052,676$ & - & -32.1 \\
\hline aal-mir-new16-3p & ATTITITGACTGTAATTTAAT & 22 & 1.245 & $1,560,997$ & $1,561,095$ & - & -26.61 \\
\hline aal-mir-new17-5p & GGGAGCGAGATTAAGGCTTGCT & 22 & 1.249 & $1,089,011$ & $1,089,093$ & - & -29.81 \\
\hline aal-mir-new18-3p & ATCCCAAGACTGCGTAGCCGT & 21 & 1.292 & $1,163,558$ & $1,163,655$ & + & -42.4 \\
\hline aal-mir-new19-5p & CGAATTTGACACTAGAGCGG & 21 & 1.299 & 707,787 & 707,872 & - & -29.5 \\
\hline aal-mir-new20-3p & GTCCCTCTGGCGCAGCGGATAGCG & 24 & 1.32 & 579,217 & 579,298 & - & -37.5 \\
\hline aal-mir-new21-3p & TAAGTGCGCTGAAGACATCA & 20 & 1.379 & 448,733 & 448,811 & + & -51.82 \\
\hline aal-mir-new22-5p & AACGGTCTAGGGTTCATGTCC & 21 & 1.389 & 711,000 & 711,091 & + & -30.4 \\
\hline aal-mir-new23-5p & AAATTTGACACTAGAGCGGG & 21 & 1.412 & 335,537 & 335,632 & - & -48.1 \\
\hline aal-mir-new24-3p & ACGATGAGGATGATGATGGTG & 21 & 1.457 & 821,927 & 822,021 & + & -31.16 \\
\hline aal-mir-new25-5p & GGGGGAAATCCTGTACGCTGTATG & 24 & 1.51 & $2,008,649$ & $2,008,731$ & + & -33.4 \\
\hline aal-mir-new26-5p & TTGGCATAAGGACGTTTGGCA & 21 & 1.526 & 450,600 & 450,680 & + & -26.7 \\
\hline aal-mir-new27-5p & GAATTTTGACACTAGAGCAGG & 21 & 1.57 & 59,966 & 60,061 & + & -44.2 \\
\hline aal-mir-new28-3p & CTGAAGAACTTTGCCGAAGAC & 21 & 1.576 & 275,303 & 275,389 & + & -30.6 \\
\hline aal-mir-new29-5p & ATTAGAATGTGGAATCTGTTIT & 22 & 1.62 & 576,218 & 576,309 & - & -32.2 \\
\hline aal-mir-new30-5p & TGGGTATITCGGAACGGGCT & 21 & 1.64 & $1,822,262$ & $1,822,351$ & - & -28.7 \\
\hline aal-mir-new31-3p & CGGAATTCCAACTGATATCCA & 21 & 1.68 & $2,729,339$ & $2,729,428$ & - & -35.25 \\
\hline aal-mir-new32-5p & CAATTITGACACTAGAGCGGG & 21 & 1.784 & 432,120 & 432,215 & - & -50.9 \\
\hline aal-mir-new33-5p & GGGAGCGAGATTAAGGCTTG & 20 & 1.249 & $1,089,011$ & $1,089,093$ & - & -29.81 \\
\hline aal-mir-3960-5p & GGCGGCGGCGGAGGTGGAGGT & 21 & 1.506 & 579,623 & 579,702 & + & -53.3 \\
\hline
\end{tabular}

Abbreviation: MFE minimum free energy

same time points by its inhibitor (Fig. 5c). The relative expression of DENV-2 significantly increased in the mimic-transfected group (2.05 times; $t_{(2)}=6.406, P=$ 0.024) compared with its expression in the $\mathrm{NCm}$ group, and it decreased in the inhibitor-transfected group by 0.28 times $\left(t_{(2)}=-7.727, P=0.016\right)$ compared with its expression in the $\mathrm{NCi}$ group (Fig. $5 \mathrm{~d}$ ). Titres in the supernatant from the mimic groups were higher than those from the $\mathrm{NCm}$ group $\left(t_{(2)}=6.36\right.$, $P=0.024)$. Furthermore, the cytopathic effect (CPE) of DENV-2 on C6/36 cells was significantly greater in cells transfected with the aal-miR-4728-5p mimic than in those transfected with $\mathrm{NCm} 4$ days postinoculation (Fig. 5a, b). Collectively, these results show that aal-miR-4728-5p may play an important role in DENV infections in Ae. albopictus. 


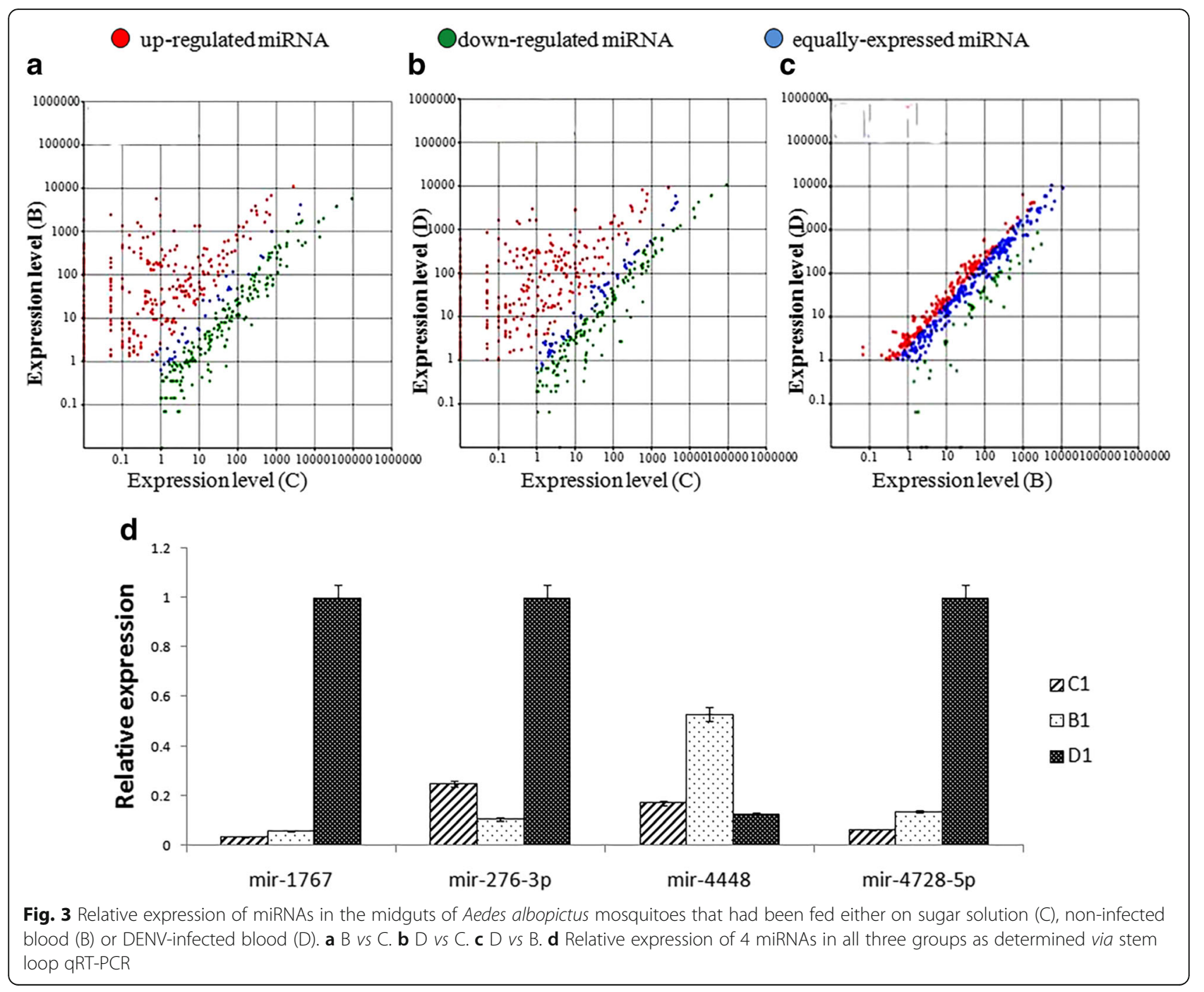

\section{Discussion}

The present study provides the first verification of six miRNAs in Ae. albopictus: aal-miR-1174 (GenBank KY062161), aal-miR-2951 (GenBank KY062162), aal-miR-956, aal-miR4728-5p (GenBank KY062160), aal-miR-1767 (GenBank KY062157) and aal-miR-4448 (GenBank KY062159). We did not detect the five previously reported Ae. albopictus miRNAs: aal-mir-286, aal-miR-315, aal-miR-309, aal-miR929 and aal-mir-971. However, aal-mir-286 was only detected in embryos, aal-miR-315is highly expressed in embryos but not at other life-stages, and aal-miR-309, aalmiR-929 and aal-mir-971 are only weakly expressed in adult mosquitoes [37]. This suggests that these miRNAs are probably tissue and life-stage specific and are not expressed in the midgut of adult female Ae. albopictus. We found marked changes in miRNA expression following the ingestion of ablood meal compared with the changes in response to a sugar solution. MiRNAs upregulated following the ingestion of a blood meal $(30 / 173 ; 17.3 \%)$ are related to the digestion of blood and the resistance to invasion by blood-borne pathogens. Interestingly, most miRNAs in the midguts of Ae. albopictus(96/173, 55.5\%) were downregulated $24 \mathrm{~h}$ after the ingestion of a blood meal, including the majority of those that had been highly expressed. For example, the expression levels of miR-956-3p and miR-184in Group B were one order of magnitude lower than in Group C. This phenomenon has also been observed in other mosquito species; for example, the expression of miR-275 in the fat body of Ae. aegypti has been shown to first decline $24 \mathrm{~h}$ post-ingestion of a blood meal ( $\mathrm{pbm})$ and then increase $48 \mathrm{~h}$ pbm [38]. In our experiment, although miR275 was downregulated $24 \mathrm{~h} \mathrm{pbm}$ it was still expressed at a relatively high level. In contrast, the expression of miR-275 in the midgut of Ae. aegypti resulted in very low (reads < 10) and showed little increase $24 \mathrm{~h} \mathrm{pbm}$. With the exception of miR-275, we found that 7 miRNAs, miR-1175, miR184, miR-281, miR-283, miR-317 and miR-34, showed the opposite trend, i.e. they were downregulated in the midgut 
Table 5 Significantly modulated miRNAs that displayed $a<-2$ - or $>2$-fold difference in expression between the midguts of mosquitoes that had ingested a DENV-2-infected blood meal (D) and those that had ingested a regular blood meal (B)

\begin{tabular}{|c|c|c|c|c|c|c|}
\hline miR-name & Reads (B) & Reads (D) & Normalized reads (B) & Normalized reads (D) & Fold-change & $P$-value \\
\hline miR-1000-5p & 20 & 159 & 1.39 & 10.09 & 2.9 & 0.00001 \\
\hline miR-15b & 373 & 2404 & 25.94 & 152.52 & 2.6 & 0.00001 \\
\hline miR-1767 & 5149 & 21,657 & 358.14 & 1373.98 & 2.0 & 0.00001 \\
\hline miR-1891 & 1 & 31 & 0.07 & 1.97 & 4.8 & 0.00001 \\
\hline miR-193-5p & 764 & 3934 & 53.14 & 249.58 & 2.2 & 0.00001 \\
\hline miR-276-3p & 1394 & 9020 & 96.96 & 572.25 & 2.6 & 0.00001 \\
\hline miR-375 & 23 & 1 & 1.60 & 0.06 & -4.7 & 0.00001 \\
\hline miR-3811e-5p & 577 & 2895 & 40.13 & 183.67 & 2.2 & 0.00001 \\
\hline miR-4448 & 42,962 & 7183 & 2988.27 & 455.71 & -2.7 & 0.00001 \\
\hline miR-4728-5p & 444 & 2519 & 30.88 & 159.81 & 2.4 & 0.00001 \\
\hline miR-6134 & 777 & 3757 & 54.05 & 238.35 & 2.1 & 0.00001 \\
\hline miR-622 & 13,931 & 100,817 & 968.99 & 6396.11 & 2.7 & 0.00001 \\
\hline miR-927-3p & 14 & 61 & 0.97 & 3.87 & 2.0 & 0.00001 \\
\hline miR-92a & 183 & 776 & 12.73 & 49.23 & 2.0 & 0.00001 \\
\hline miR-989-3p & 149 & 42 & 10.36 & 2.66 & -2.0 & 0.00001 \\
\hline miR-998 & 205 & 936 & 14.26 & 59.38 & 2.1 & 0.00001 \\
\hline
\end{tabular}

of Ae. albopictus but were upregulated in the midgut of $A e$. aegypti at the same time point pbm [39]. MiR-275 expression is upregulated in the body of Ae. aegypti pbm and has been found to be indispensable for blood digestion and egg development, but its function in Ae. albopictus is uncertain. We found that miR-375 was expressed in the midgut before the ingestion of a blood meal (15 reads), and its expression increased $24 \mathrm{~h}$ PBM (23 reads). In Ae. aegypti, miR-375 was not expressed in the midgut and was only detected in the bodies of blood-fed females. This difference between these two Aedes mosquitoes may partly reflect differences in the expression patterns and roles of different molecular factors.
Compared with expression in Group B, most of the miRNAs that were differentially expressed in Group D, such as miR-1175, miR-276, miR-317, miR-34-5p, miR1767 and miR-375, were upregulated, although the expression of miR-989 decreased to a very low level. A recent study on infection of Ae. aegypti by DENV-2 indicates that among the 31 miRNAs with relatively marked differences in expression levels between Groups D and B, only four, miR-34-3p, miR-5119-5p, miR-87$5 \mathrm{p}$, miR-988-5p, were upregulated; the remaining 27 , including miR-1175, miR-276-5p, miR-281, miR-2945, miR-317 and miR-33-5p, were downregulated in Group

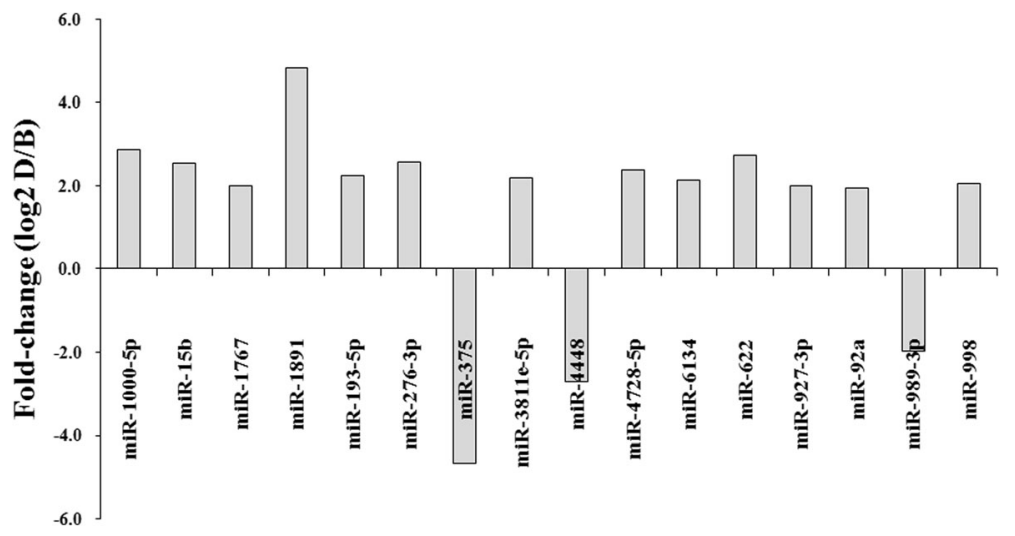

Fig. 4 Relative expression of miRNAs with more than 2-fold difference in expression between the midguts of DENV-2-infected and uninfected mosquitoes. "Fold-change"is the magnitude of change in miRNA expression in the DENV-2-infected group relative to that in the uninfected group (fold-change $=\log _{2}$ ratio) 
a

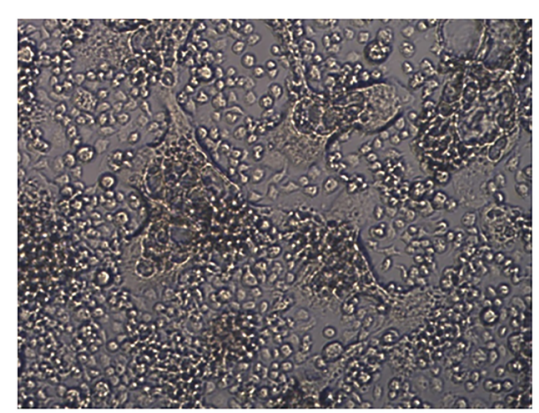

C

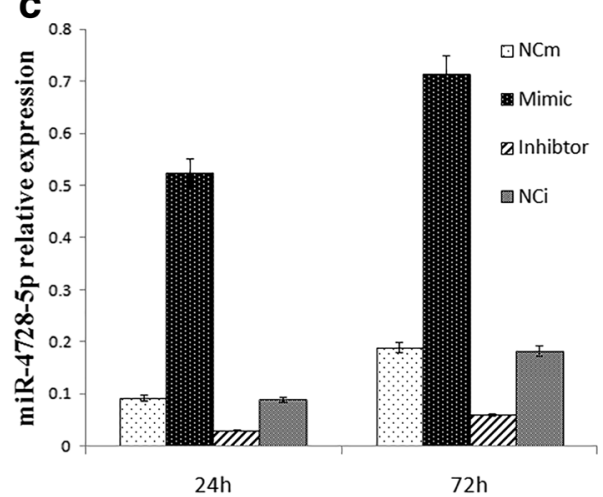

b

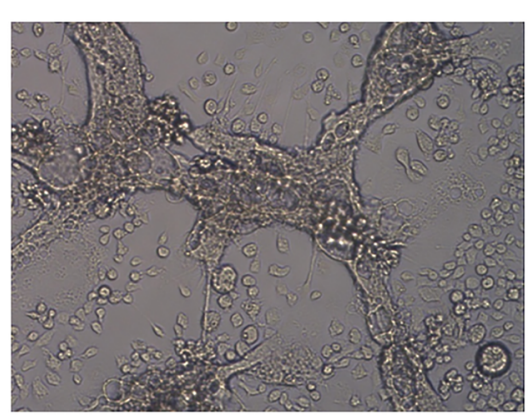

d

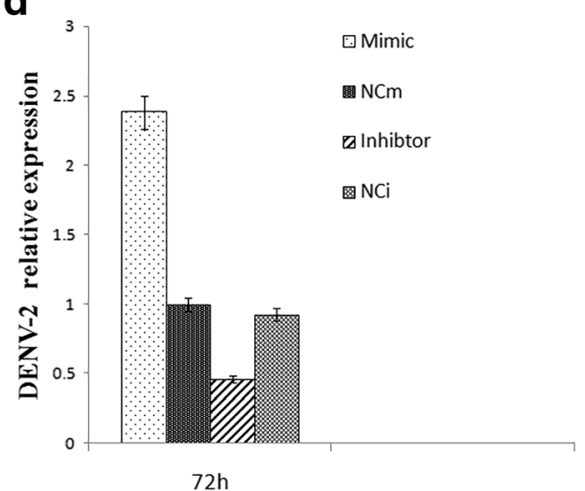

Fig. 5 Effects of aal-miR-4728-5p on the replication of DENV-2 in C6/36 cells. a Cytopathic effect (CPE) of DENV-2 in C6/36 cells that had been transfected with an aal-miR-4728-5p inhibitor. b CPE in C6/36 cells that had been transfected with an aal-miR-4728-5p mimic. c Relative expression of aal-miR-4728$5 p$ in C6/36 cells $24 \mathrm{~h}$ and $72 \mathrm{~h}$ post-transfection with the mimic, inhibitor and negative controls. $\mathbf{d}$ Relative DENV-2 expression levels in C6/36 cells after transfection with an aal-miR-4728-5p mimic or inhibitor. Relative expression levels of aal-miR-4728-5p and DENV-2 were determined via qRT-PCR and calculated using the $2^{-\Delta \Delta C T}$ method. Abbreviations: NCm, negative control for the aal-miR-4728-5p mimic; NCi, negative control for the aal-miR-4728-5p inhibitor

D compared with their expression in Group C [21]. Another study found that the expression of miR-34, miR1174 and miR-1175 in midgut epithelial cells of $A n$. gambiae decreased, whereas that of miR-989 increased four-fold 24-48 h after Plasmodium infection [29]. These results indicate that some miRNAs, such as miR1175, miR-34 and miR-989, may have different functions in different species; for example, miR-1175, miR-276 and miR-317 display contrary trends in their expression in Ae. albopictus and Ae. aegypti, and the corresponding molecular mechanisms may also be different in each species.

Among the miRNAs listed in Table 5, miR-375 enhances DENV infections in Ae. aegypti Aag2 cells, which suggests that it may be involved in DENV infection in this species [20]. In this study, the expression level of miR-375 in the midgut was too low to suggest that it plays a role in the process of DENV infection in the midgut of Ae. albopictus. Further more, miR-275 expression was upregulated in Group $\mathrm{D}$ by a ratio of 2.67 (1.37-fold) compared with its expression in Group B. This result, together with its function in Ae. aegypti, suggests that this miRNA may play a role in DENV infections in the midgut of Ae. albopictus.

We also found that many miRNAs that were expressed at very low levels, such as miR-252, showed significant differences in expression between Groups D and B. Although miR-252was upregulated in Group D, its expression levels were very low, suggesting that it is unlikely to play a role in midgut infections. In another study, miR252 was found to be abundantly expressed in adult female Ae. albopictus and was downregulated 7 days after an intrapleural injection of DENV-2. Furthermore, a transient transfection assay showed that miR-252 inhibited the replication of DENV-2 in C6/36 cells [40]. These differences suggest that this miRNA may have different expression patterns in different organs in Ae. albopictus. However, it is also possible that the change in miRNA expression induced by oral infections could be different from those induced by intrapleural injections, which is not the natural pathway of infection.

Aal-miR-4728-5p, a conserved miRNA that was newly discovered in Ae. albopictus, was expressed in the midgut before the ingestion of a blood meal (109 reads), but its expression subsequently increased PBM in infected 
midguts (2519 reads) compared with its expression in uninfected midguts (444 reads). MiR-4728-5p is also expressed in humans, where it is involved in tumourigenesis [41]. There has been no previous research on the role of this miRNA in DENV infections, but our transient transfection experiments indicate that it enhances the replication of DENV-2 in C6/36 cells. We plan to conduct similar experiments on other miRNAs that displayed significant differences in expression between infected and uninfected mosquitoes. This work should improve our understanding of the miRNAs involved in the process of midgut infection by the DENV.

In summary, this study provides the first information on miRNAs in Ae. albopictus midgut and suggests potential avenues for further research on the role of these miRNAs during DENV infections in Ae. albopictus. In the absence of the complete Ae. albopictus genome, the majority of the miRNAs we found remain unidentified. Mapping our RNA libraries to the Ae. albopictus genome, when this becomes available, will help us determine the structure and function of all the reads obtained. A better understanding of the mechanisms responsible for the midgut infection barrier could lead to new insights in mosquito biology and novel approaches for combating mosquito-borne infectious diseases.

\section{Conclusion}

The present study provides the first information on microRNAs expressed in the midgut of Ae. albopictus and describes species-specific changes in their expression levels following DENV-2 infection. It was confirmed that six miRNAs, aal-miR-1174, aal-miR-2951, aal-miR956, aal-miR-4728-5p, aal-miR-1767 and aal-miR-4448, in the midguts of wild Ae. albopictus differ from those in laboratory strains. The aal-miR-4728-5p was chosen for an in vitro transient transfection assay, and the results show that this miRNA enhances DENV replication in $\mathrm{C} 6 / 36$ cells.

\section{Additional files}

Additional file 1: Table S1. Conserved miRNAs and their expression in the midgut of Ae. albopictus mosquitoes that were fed with sugar, regular blood and DENV-2-infected blood. (DOCX 34 kb)

Additional file 2: Table S2. Name, sequence, location, normalized expression of miRNAs from the midguts adult female Ae. albopictus mosquitoes. (DOCX $34 \mathrm{~kb})$

\section{Abbreviations}

aae-mir-1174: Aedes aegypti microRNA -1174 stem loop; C6/36: Aedes albopictus clone; cDNA: complementary deoxynucleic acid; Ct: Threshold cycle; DENV: Dengue virus; DENV-2: Dengue virus 2; DF: Dengue fever; DHF: Dengue haemorrhagic fever; DMEM: Dulbecco's Modified Eagle's Medium; dNTP: Deoxynucleotide triphosphate; FBS: Foetalbovine serum; miR1174: microRNA-1174; miR-1175: microRNA-1175; miRNA: microRNA; miRNAs: microRNAs; PCR: Polymerase chain reaction; RT: Reverse transcription; sRNA: Small RNA

\section{Acknowledgements}

Not applicable.

\section{Funding}

This work was funded by grants from the Infective Diseases Prevention and Cure Project of National Ministry of Public Health of China (No. 2012ZX10004219)

\section{Availability of data and material \\ The aal-miR-1767 sequence is available in the adult Ae. albopictus database (https://www.ncbi.nIm.nih.gov/KY062157/) \\ The aal-miR-1174 sequence is available in the adult Ae. albopictus database (https://www.ncbi.nlm.nih.gov/KY062161/) \\ The aal-miR-2951 sequence is available in the adult Ae. albopictus database (https://www.ncbi.nlm.nih.gov/KY062162/) \\ The aal-miR-956-3p sequence is available in the adult Ae. albopictus database (https://www.ncbi.nlm.nih.gov/KY062163/) \\ The aal-miR-4728-5p sequence is available in the adult Ae. albopictus database (https://www.ncbi.nlm.nih.gov/KY062160/) \\ The aal-miR-4448 sequence is available in the adult Ae. albopictus database (https://www.ncbi.nlm.nih.gov/KY062159/}

\section{Authors' contributions}

JXS and TYZ contributed to the manuscript design, acquisition of data and interpretation of data. JXS wrote the manuscript, and XXG was involved in drafting the manuscript. TY, CXL, YMZ, XJZ, DX and MHZ carried out the laboratory work and performed the statistical analysis. TYZ made the decision to submit the manuscript for publication. All authors read and approved the final manuscript.

\section{Competing interests}

The authors declare that they have no competing interests.

\section{Consent for publication}

Not applicable.

\section{Ethics approval}

All vertebrate animals were housed and handled in strict accordance with the guidelines of the institutional and national Committees for Animal Use and Protection. The experimental procedures on mice were approved by the Animal Experiment Ethics Committee of the Beijing Institute of Microbiology and Epidemiology.

\section{Author details}

${ }^{1}$ State Key Laboratory of Pathogen and Biosecurity, Beijing Institute of Microbiology and Epidemiology, Beijing 100071, People's Republic of China ${ }^{2}$ Center for Disease Control and Prevention of Guangzhou Military Region, Guangzhou 510507, People's Republic of China.

Received: 12 July 2016 Accepted: 2 January 2017

Published online: 03 February 2017

\section{References}

1. Rigau-Pérez JG, Clark GG, Gubler DJ, Reiter P, Sanders EJ, Vorndam AV. Dengue and dengue haemorrhagic fever. Lancet. 1998;352:971-7.

2. Gould EA, Solomon T. Pathogenic flaviviruses. Lancet. 2008;371:500-9.

3. Kyle JL, Harris E. Global spread and persistence of dengue. Annu Rev Microbiol. 2008:62:71-92

4. Gubler DJ. Surveillance for dengue and dengue hemorrhagic fever. Bull Pan Am Health Organ. 1989;23:397-404.

5. Robert MA, Okamoto K, Lloyd AL, Gould F. A reduce and replace strategy for suppressing vector-borne diseases: insights from a stochastic, spatial model. PLoS One. 2013:8:e81860.

6. Beaty BJ. Genetic manipulation of vectors: a potential novel approach for control of vector-borne diseases. Proc Natl Acad Sci USA. 2000;97:10295-7.

7. Aksoy S, Maudlin I, Dale C, Robinson AS, O'Neill SL. Prospects for control of African trypanosomiasis bytsetse vector manipulation. Trends Parasitol. 2001; 17:29-35.

8. Alphey L. Malaria control with genetically manipulated insect. Nature. 2002;415:702 
9. Hardy JL, Houk EJ, Kramer LD, Reeves WC. Intrinsic factors affecting vector competence of mosquitoes for arboviruses. Ann Rev Entomol. 1983;28:229-62.

10. William IV, Bennett KE, Gorrochótegui-Escalante N, Barillas-Mury CV, Fernández-Salas I, de Lourdes Muñoz MA, et al. Flavivirus susceptibility in Aedes aegypti. Arch Med Res. 2002;33:379-88.

11. Woodring JL, Higgs S, Beaty BJ. Natural cycles of vector-borne pathogens. In: Beaty BJ, Marquardt WC, editors. The biology of disease vectors. University Press of Colorado; Niwot: 1996. pp. 51-72.

12. Khoo CC, Piper J, Sanchez-Vargas I, Olson KE, Franz AW. The RNA interference pathway affects midgut infection-and escape barriers for Sindbis virus in Aedes aegypti. BMC Microbiol. 2010;10:130.

13. Bartel DP. MicroRNAs: genomics, biogenesis, mechanism, and function. Cell. 2004:116:281-97.

14. Plasterk RH. Micro RNAs in animal development. Cell. 2006;124:877-81.

15. Lee RC, Feinbaum RL, Ambros V. The C. elegans heterochronic gene lin- 4 encodes small RNAs with antisense complementarity to lin-14. Cell. 1993;75: 843-54.

16. Esau CC, Monia BP. The rapeutic potential for microRNAs. Adv Drug Deliv Rev. 2007:59:101-14.

17. Mead EA, Li M, Tu Z, Zhu J. Translational regulation of Anopheles gambiae mRNAs in the midgut during Plasmodium falciparum infection. BMC Genomics. 2012;13:366.

18. Winter J, Diederichs S. Argonaute proteins regulate microRNA stability. RNA Biol. 2011;8:1149-57.

19. Franz AW, Sanchez-Vargas I, Adelman ZN, Blair CD, Beaty BJ, James AA, Olson KE. Engineering RNA interference-based resistance to dengue virus type 2 in genetically modified Aedes aegypti. Proc Natl Acad Sci USA. 2006; 103:4198-203.

20. Hussain M, Walker T, O'Neill SL, Asgari S. Blood meal induced microRNA regulates development and immune associated genes in the Dengue mosquito vector, Aedes aegypti. Insect Biochem Mol Biol. 2013;43:146-52.

21. Campbell $\mathrm{CL}$, Harrison T, Hess AM, Ebel GD. MicroRNA levels are modulated in Aedes aegypti after exposure to Dengue-2. Insect Mol Biol. 2014;23:132-9.

22. Sota T, Mogi M. Interspecific variation in desiccation survival time of Aedes (Stegomyia) mosquito eggs is correlated with habitat and egg size. Oecologia. 1992;90:353-8.

23. Bäck AT, Lundkvist Å. Dengue viruses - an overview. Infect Ecol Epidemiol. 2013:3:1-21

24. Mitchell C, Miller B, Gubler D. Vector competence of Aedes albopictus from Houston, Texas, for dengue serotypes 1 to 4, yellow fever and Ross River viruses. J Am Mosquito Control Assoc. 1987;3:460-5.

25. Johnson BW, Chambers TV, Crabtree MB, Filippis A, Vilarinhos PT, Resende MC, et al. Vector competence of Brazilian Aedes aegypti and Ae. albopictus for a Brazilian yellow fever virus isolate. Trans R Soc Trop Med Hyg. 2002;96:611-3.

26. Lambrechts L, Scott TW, Gubler DJ. Consequences of the expanding global distribution of Aedes albopictus for dengue virus transmission. PLoS Negl Trop Dis. 2010;4:e646.

27. Scott JC, Brackney DE, Campbell CL, Bondu-Hawkins V, Hjelle B, Ebel GD, et al. Comparison of Dengue virus type 2-specific small RNAs from RNA interference-competent and -incompetent mosquito cells. PLoS Negl Trop Dis. 2010:4:e848.

28. Bushati N, Cohen SM. MicroRNA functions. Annu Rev Cell Dev Biol. 2007;23: 175-205.

29. Winter F, Edaye S, Hüttenhofer A, Brunel C. Anopheles gambiae miRNAs as actors of defence reaction against Plasmodium invasion. Nucl Acids Res. 2007;35:6953-62

30. Zhang M, Zheng X, Wu Y, Gan M, He A, Li Z, et al. Quantitative analysis of replication and tropisms of dengue virus type 2 in Aedes albopictus. Am J Trop Med Hyg. 2010;83:700.

31. Schmittgen TD, Livak KJ. Analyzing real-time PCR data by the comparative CT method. Nat Protoc. 2008;3:1101-8.

32. Audic S, Claverie JM. The significance of digital gene expression profiles. Genome Res. 1997;7:986-95.

33. Skalsky RL, Vanlandingham DL, Scholle F, Higgs S, Cullen BR. Identification of microRNAs expressed in two mosquito vectors, Aedes albopictus and Culex quinquefasciatus. BMC Genomics. 2010;11:119.

34. Gu J, Hu W, Wu J, Zheng P, Chen M, James AA, et al. miRNA genes of an invasive vector mosquito, Aedes albopictus. PLoS One. 2013;8:e67638.

35. Zheng P, Wu J, Gu J, Tu Z, Chen X. Isolation, identification and analysis of the expression profile of miRNAs in Aedes albopictus. J South Med Uni. 2010; 30:677-80.
36. Ruby JG, Stark A, Johnston WK, Kellis M, Bartel DP, Lai EC. Evolution, biogenesis, expression, and target predictions of a substantially expanded set of Drosophila microRNAs. Genome Res. 2007;17:1850-64.

37. Gratz N. Critical review of the vector status of Aedes albopictus. Med Vet Entomol. 2004;18:215-27.

38. Bryant B, Macdonald W, Raikhel AS. microRNA miR-275 is indispensable for blood digestion and egg development in the mosquito Aedes aegypti. Proc Natl Acad Sci USA. 2010;107:22391-8.

39. Li S, Mead EA, Liang S, Tu Z. Direct sequencing and expression analysis of a large number of miRNAs in Aedes aegypti and a multi-species survey of novel mosquito miRNAs. BMC Genomics. 2009:10:581.

40. Yan H, Zhou Y, Liu Y, Deng Y, Puthiyakunnon S, Chen X. MiR-252 of the Asian tiger mosquito Aedes albopictus regulates dengue virus replication by suppressing the expression of the dengue virus envelope protein. J Med Virol. 2014;86:1428-36.

41. Persson H, Kvist A, Rego N, Staaf J, Vallon-Christersson J, Luts L, et al. Identification of new microRNAs in paired normal and tumor breast tissue suggests a dual role for the ERBB2/Her2 gene. Cancer Res. 2011;71:78-86.

\section{Submit your next manuscript to BioMed Central and we will help you at every step:}

- We accept pre-submission inquiries

- Our selector tool helps you to find the most relevant journal

- We provide round the clock customer support

- Convenient online submission

- Thorough peer review

- Inclusion in PubMed and all major indexing services

- Maximum visibility for your research

Submit your manuscript at www.biomedcentral.com/submit
Biomed Central 